\title{
Magnetization field-dependences and the "exchange bias" in ferro/antiferromagnetic systems. I. Model of a bilayer ferromagnetic
}

Cite as: Low Temp. Phys. 35, 476 (2009); https://doi.org/10.1063/1.3151994

Published Online: 15 July 2009

A. G. Grechnev, A. S. Kovalev, and M. L. Pankratova

ARTICLES YOU MAY BE INTERESTED IN

Field dependences of the magnetization and exchange bias in ferro/antiferromagnetic systems. II. Continuum model of a ferromagnetic layer

Low Temperature Physics 35, 526 (2009); https://doi.org/10.1063/1.3168638

LOW TEMPERATURE TECHNIQUES OP『\|CAM CAVITV PHYSICS MITIGATING THERMAL \& VIBRATIONAL NOISE

\section{DOWNLOAD THE WHITE PAPER}




\title{
Magnetization field-dependences and the "exchange bias" in ferro/antiferromagnetic systems. I. Model of a bilayer ferromagnetic
}

\author{
A. G. Grechnev and A. S. Kovalev ${ }^{\text {a) }}$ \\ B. I. Verkin Institute for Low-Temperature Physics and Engineering of the National Academy of Sciences of \\ Ukraine, pr. Lenina 47, Kharkov 61103, Ukraine
}

\section{L. Pankratova}

V. N. Karazin Kharkov National University, pl. Svobody 4, Kharkov 61107, Ukraine

(Submitted February 10, 2009; revised February 18, 2009)

Fiz. Nizk. Temp. 35, 603-612 (2009)

\begin{abstract}
A qualitative model explanation of the experimentally obtained field dependences of the magnetization in ferro- and antiferromagnetic media in contact with one another is proposed. In this model a thin ferromagnetic (FM) film on an antiferromagnetic (AFM) substrate consists of only two ferromagnetic layers. This is the simplest model which admits a spatially nonuniform FM state. In this exactly solvable model it shown that a range of fields exists where a stable collinear (canted) structure of the FM subsystem obtains. This structure corresponds to inclined sections of the field dependence $M(H)$ of the magnetization which are not associated with the kinetics of the magnetization reversal process. In the model proposed, for systems with large easy-plane anisotropy the magnetization reversal process with "exchange bias" taken into account is strictly symmetric as a function of the field provided that the additional weak FM anisotropy in the easy plane is neglected. When this anisotropy in the easy plane is taken into account hysteresis appears in the magnetization curve and the field dependence $M(H)$ becomes asymmetric.
\end{abstract}

(C) 2009 American Institute of Physics. [DOI: 10.1063/1.3151994]

\section{INTRODUCTION}

Interest in magnetic multilayer structures is motivated by their many applications, both already and not yet realized, in magneto- and spin electronics. Examples are highly sensitive magnetic-field sensors and magnetic writing and storage of information. There is great interest in studying phase transitions, spin structure, scaling (qualitative change of the properties), and proximity effects as functions of the dimension of the materials. $\mathrm{Bi}$ - and multilayer magnetic structures consist of alternating layers with different magnetic properties. Multilayer structures with alternating ferro- and antiferromagnetic layers are especially interesting. These objects are promising in connection with the study and application of the phenomenon of giant magnetoresistance. They are of interest from the theoretical standpoint as systems where media with different spatial symmetry are contiguous. ${ }^{1-3}$

One of the most intriguing phenomena in the physics of low-dimensional magnetic systems is the phenomenon of exchange bias (EB) or exchange anisotropy. ${ }^{4-6}$ This effect, which Mieklejohn and Bean discovered in 1956 for oxidized cobalt $(\mathrm{CoO})$ microparticles,${ }^{4}$ consists of a displacement of the hysteresis loop in the magnetization as a function of the external magnetic field $M=M(H)$ from the symmetric position relative to the point $H=0$. The EB phenomenon arises in systems with a ferromagnet (FM)/antiferromagnet (AFM) interface when the system is cooled below the Néel temperature $T_{N}$ of the AFM (which should be less than the Curie temperature $T_{C}$ of the FM) in an external magnetic field. Although the effect was first observed for FM microparticles coated with an AFM film, layered FM/AFM systems are most convenient for studying the effect and for technical applications. The EB phenomenon is used in the read/write heads of modern hard disks to fix the direction of magnetization at $H=0$.

Qualitatively, the effect consists of the following. At temperatures above $T_{N}$ but below $T_{C}$ the AFM is a magnetically disordered state and the FM is in an ordered state. As temperature decreases below $T_{N}$ in the presence of a field $H$ the antiferromagnet becomes ordered, and the exchange bias through the interface gives an effective magnetic field $H_{\text {bias }}$ which displaces the hysteresis loop $M=M(H)$. This is a surface effect, which decreases with increasing thickness of the FM layer as $H_{\text {bias }} \sim 1 / L_{F}$, where $L_{F}$ is the thickness of the FM layer. ${ }^{5,6}$ The simplest theoretical model of the phenomenon, ${ }^{4}$ which is predicated on uniform magnetization of the FM layer, gives an expression for the exchange bias $H_{\text {bias }}=J_{0} S_{A} S_{F} / L_{F} M_{F}$, where $S_{A}$ and $S_{F}$ are the AFM and FM magnetic moments, respectively, $M_{F}$ is the magnetization of the FM (for lattice constant along the FM/AFM interface), and $J_{0}$ is the exchange through the interface. Experiments show that this expression is greater than $H_{\text {bias }}$ by two to four orders of magnitude, if it is assumed that $J_{0}$ is of the same order of magnitude as the exchange interaction inside the FM layer. In addition, we note that the FM/AFM interface can be compensated (the total magnetic moment of the near-surface AFM layer is zero) or uncompensated. ${ }^{1-3}$ It would be natural to expect that the EB effect arises only for uncompensated interfaces. However, in reality, $H_{\text {bias }}$ is of the same order of magnitude for both types of interfaces. Many theoretical models studying its different aspects were proposed for the EB effect from the moment it was discovered. ${ }^{7}$ For example, Refs. 1 and 8-14 examine the possibility of the formation of domain walls (DWs) or incomplete domain walls parallel to 

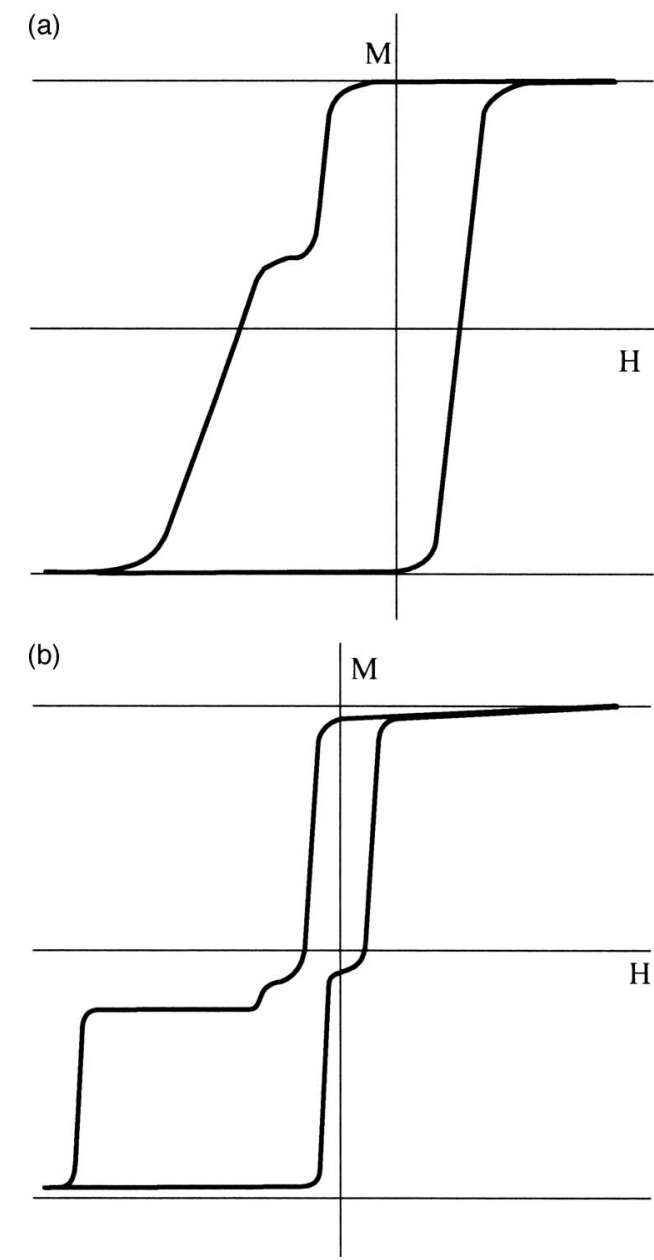

FIG. 1. Experimental magnetization-versus-field curves from Ref. 21 (a) and Ref. 22 (b). The asymmetry of the field shift and a "small pedestal" for $H<0$ are clearly seen.

the interface; Refs. 14-16 studied the role of defects of the interface and of the domain and polycrystalline structure of magnets; and, Refs. 10, 11, and 17 attempted to explain the nature of EB in systems with a compensated interface. In spite of the large number of works in existence, there is still no exhaustive theory of the EB phenomenon.

Recently, new particularities of the exchange bias phenomenon have been found experimentally. Specifically, the displaced hysteresis loop becomes asymmetric (i.e. $\left.M\left(2 H_{\text {bias }}-H\right) \neq-M(H)\right)$ and additional steps appear in it. $^{18-22}$ The slope of the magnetization curve is different on different sections; this could be due to different spin-flip kinetics in different magnetic configurations. Exaggerated diagrams of magnetization reversal in these cases are presented in Fig. 1. These diagrams correspond qualitatively to the diagrams presented in Refs. 18-22. The "small pedestals" present in the field dependence draw attention. The field dependence $M(H)$ presented in Fig. 1b is also interesting. Two regions can be discerned here. The top part of the hysteresis loop (for positive values of the field) is almost symmetric with respect to the field; this is characteristic for ordinary hysteresis. A wide, field-shifted, hysteresis loop is seen in the bottom part of the dependence $M(H)$. The presence of "small pedestals" and field-separated sections of the dependence $M(H)$ indicates the possible existence of unusual, additional, stationary states which differ from the completely magnetically reversed states. Different theories ${ }^{23-25}$ developed to explain the asymmetry of the hysteresis loop deploy additional assumptions, such as the presence of a nonzero angle between the direction of the FM magnetization and the AFM anisotropy axis or the presence of higher-order terms in the exchange interaction through the FM/AFM boundary. ${ }^{23,24} \mathrm{On}$ the other hand, it is shown in Refs. 10-13 that for sufficiently thick FM layers a portion of the anisotropy in $M(H)$ arises naturally in the simplest models of the EB phenomenon.

The present article examines a model proposed in Refs. 10-13 for the FM/AFM interface. In this model the AFM is a magnetically rigid material, i.e. the magnetic moments of the AFM are fixed. The FM moments are not fixed and interact with an external field $H$, with one another (the exchange integral $J$ ), and with the contiguous AFM layer (exchange integral $J_{0}$ ), forming objects of the type incomplete DWs in a FM layer. Then the exchange integral $J_{0}$ must be interpreted as an effective interaction ${ }^{10-13,16}$ that takes account of the presence of defects in the interface and partial lack of compensation of the AFM boundary. Obtaining a theoretical estimate of this parameter is a quite difficult problem. ${ }^{10-13,16}$ Experiments show that $J_{0} / J \sim 10^{-2}-10^{-3}$. The present article examines a wider range of values of the ratio $J_{0} / J$. In a subsequent article we shall show that an increase of this parameter corresponds to an effective decrease of the thickness of the FM layer; this justifies studying in the present work anomalously thin films with $J_{0} / J \sim 1$. Just as in Refs. 10-13, in the main part of this work the additional easy-plane magnetic anisotropy of the FM, which results in the appearance of hysteresis, is neglected. This is because the anisotropy does not qualitatively affect the symmetry of the magnetization reversal curve or the field $H_{\text {bias }}$, which in the absence of anisotropy is determined from the condition $M\left(H_{\text {bias }}\right)=0$. The present model was studied numerically or analytically in Refs. 10-13 using a very simple approach.

Our objective in the present work is to derive and investigate exact analytic solutions of the problem in the limiting case of a ferromagnet consisting of two atomic layers, i.e. in the simplest possible model that still admits the existence of nonuniform FM states.

\section{THEORETICAL MODEL OF A BILAYER FERROMAGNET}

We shall study a simple model in which the ferromagnetic part of the system consists of only two atomic layers (in reality, the number of layers can reach several tens). The case of a single FM layer is examined in Ref. 4 and is trivial; the minimal model admitting nonuniform states of the DW type consists of two layers. The investigation of nonlinear discrete systems with a small number of degrees of freedom shows that some of their structural and dynamic properties have analogues in systems with a large number of degrees of freedom and even in systems with distributed parameters. ${ }^{26,27}$ The first system that we shall examine is a ferromagnetic subsystem with strong easy-plane anisotropy, which "packs" spins into this plane, in the absence of additional weak anisotropy in the easy plane. (In what follows "taking account of anisotropy" means taking account of the additional magnetic anisotropy in the easy plane.) For simplicity, we as- 


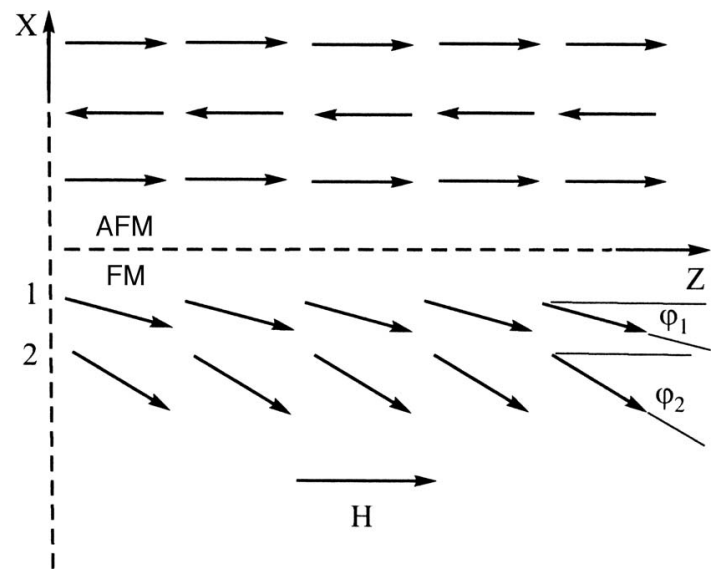

FIG. 2. Diagram of the model proposed for a bilayer ferromagnetic in contact with an uncompensated boundary of an antiferromagnet.

sume that the state is uniform along the interface. We assume that the spins in the AFM layer which is in contact with the boundary are directed along the $Z$ axis and an external magnetic field in applied in this same direction. Then the model becomes scalar and is described by two angles of rotation of the spins in the easy-plane away from the direction of the $Z$ axis for the two FM layers: $\varphi_{1}$ and $\varphi_{2}$. The AFM is assumed to be layered and the interface uncompensated, i.e. uniform. In Fig. 2 the interface between the ferro- and antiferromagnets is directed along the $Z$ axis, and the easy-plane corresponds to the $(Z X)$ plane. The energy of the FM in the system, including the interaction through the interface, is

$$
E=-J_{0} \cos \varphi_{1}-J \cos \left(\varphi_{1}-\varphi_{2}\right)-H\left(\cos \varphi_{1}+\cos \varphi_{2}\right),
$$

where $J_{0}$ is the exchange interaction through the boundary (assumed to be ferromagnetic), $J$ is the exchange between the FM layers, and $H$ is the external magnetic field. It is easy to see that this energy is invariant under the transformations $H \leftrightarrow-J_{0}-H$ and $\varphi_{1} \leftrightarrow \pi-\varphi_{2}$. Here $M\left(-J_{0}-H\right)=-M(H)$, which signifies that the EB field in this model $H_{\text {bias }}=-J_{0} / 2$, which is identical to the results of the model proposed in Ref. 4. In a field shifted by the amount $H_{\text {bias }}$ the magnetization curve is symmetric. Thus a model with two spins and no anisotropy cannot explain the asymmetry of the hysteresis loop.

The equilibrium spin configurations are determined by the system of equations $\partial E / \partial \varphi_{1}=\partial E / \partial \varphi_{2}=0$, which reduce to the following system:

$$
\begin{aligned}
& \left(H+J_{0}\right) \sin \varphi_{1}+J \sin \left(\varphi_{1}-\varphi_{2}\right)=0, \\
& H \sin \varphi_{2}-J \sin \left(\varphi_{1}-\varphi_{2}\right)=0 .
\end{aligned}
$$

These equations give a simple relation between the angles $\varphi_{1}$ and $\varphi_{2}$ :

$$
\sin \varphi_{2}=-\left(1+J_{0} / H\right) \sin \varphi_{1} .
$$

\section{FIELD DEPENDENCES OF THE MAGNETIZATION OF FERROMAGNETIC LAYERS}

The equation (3) possesses the obvious solutions $\sin \varphi_{1}=0, \quad \sin \varphi_{2}=0$,

describing collinear magnetic structures with parallel and antiparallel spin orientations, which can be conventionally represented in the form $\uparrow \uparrow\left(\varphi_{1}=\varphi_{2}=0\right), \uparrow \downarrow\left(\varphi_{1}=0, \varphi_{2}=\pi\right)$, $\downarrow \uparrow\left(\varphi_{1}=\pi, \varphi_{2}=0\right)$, and $\downarrow \downarrow\left(\varphi_{1}=\varphi_{2}=\pi\right)$, where the left-hand arrow corresponds to the direction of magnetization in the first FM layer and right-hand arrow denotes the same in the second layer; the angles are measured from the direction of magnetization in the adjoining AFM layer. The respective energies of these states are: $E_{\uparrow \uparrow}=-J_{0}-J-2 H, E_{\uparrow \downarrow}=-J_{0}-J$, $E_{\downarrow \uparrow}=J_{0}+J$, and $E_{\downarrow \downarrow}=J_{0}-J+2 H$. For $J>J_{0}$ the energy minimum corresponds for $H>H_{\text {bias }}=-J_{0} / 2$ to the state $\uparrow \uparrow$ with total magnetization $M=2$ and for $H<H_{\text {bias }}$ to the state $\downarrow \downarrow$ with $M=-2$. The dependence $M=M(H)$ for collinear states is a step function with the step shifted to negative fields by the amount $H_{\text {bias. }}$. For the opposite inequality $J<J_{0}$ (we shall examine this case also, though in any real situation the opposite and stronger condition $J_{0} \ll 2 J$ ordinarily holds) there are three regions of collinear structures with two critical fields $H_{1}=-J_{0}+J<H_{2}=-J$. In the region $H<H_{1}$ the phase with the lowest energy is the $\downarrow \downarrow$ phase with magnetization $M=-2$; in the region $H_{1}<H<H_{2}$ the comparable phase is $\uparrow \downarrow$ with $M=0$ ("antiparallel"); and, in the region $H>H_{2}$ the comparable phase is $\uparrow \uparrow$ with $M=2$. In this case a "small pedestal" with $M=0$, shifted to negative fields by the amount $H_{\text {bias }}=J_{0} / 2$, can be present in the field dependence $M(H)$. The $\downarrow \uparrow$ phase for $J_{0}>0$ is not realized. However, as we shall show, the antiparallel phase $\uparrow \downarrow$ is stable in a narrower field interval and only if $J<J_{0} / 2$.

This is because collinear structures do not exhaust all solutions of Eq. (3): noncollinear phases with the magnetization vectors tilted away from the direction of the magnetization field are also possible. The nonuniform states with turning of the magnetization which are being studied here are not domain walls, but structurally they are reminiscent of the latter and they exist only in an external magnetic field. For further analysis it is convenient to introduce a dimensionless external magnetic field shifted relative to zero field by $H_{\text {bias }}$ and the dimensionless exchange interaction parameter

$$
h=2 H / J_{0}+1, \quad I=2 J / J_{0} .
$$

In this notation the magnetization jump occurs at $h=0$ and the anticollinear phase arises for $I<1$ and occupies a symmetric region $I-1<h<1-I$. The distribution of the magnetic moments in the noncollinear structure has the following form:

$$
\cos \varphi_{1,2}=\frac{4 I^{2} h \pm\left(1-h^{2}\right)^{2}}{2 I\left(1-h^{2}\right)(1 \pm h)} .
$$

Note that these expression possess the symmetry $\cos \varphi_{1}(h)$ $=-\cos \varphi_{2}(-h)$, which is a trivial consequence of the abovementioned symmetry of the problem. The dependence of the magnetization of the ferromagnetic part on the external field assumes the form

$$
M(h)=\cos \varphi_{1}(h)+\cos \varphi_{2}(h)=h \frac{4 I^{2}-\left(1-h^{2}\right)^{2}}{I\left(1-h^{2}\right)^{2}} .
$$

It is evident that this function is asymmetric in the field $h$ (shifted): $M(-h)=-M(h)$. It should be kept in mind that the 
(a)

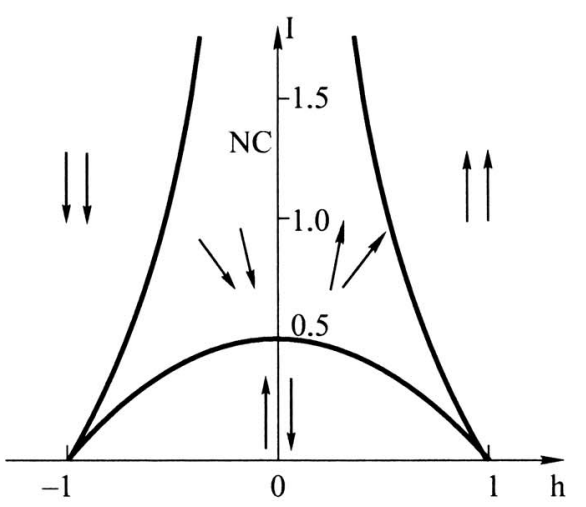

(b)

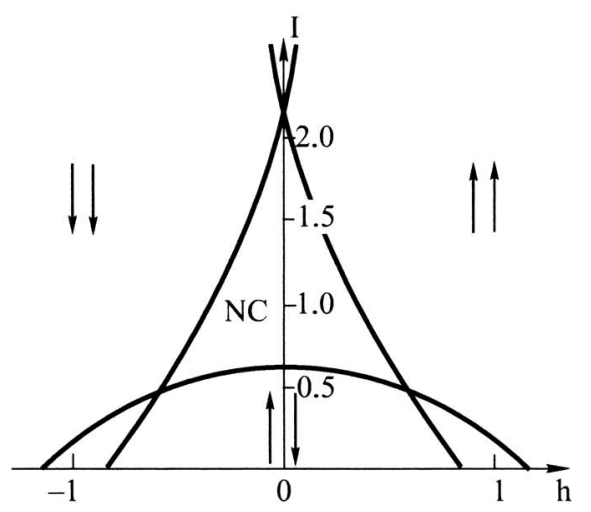

(c)

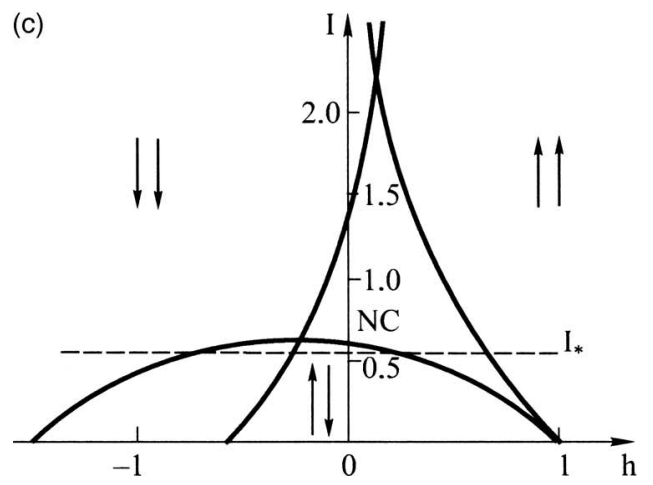

FIG. 3. Phase diagram of a bilayer model of a ferromagnet in the variables $(h, I)$. The regions of stability of the collinear phases $\uparrow \uparrow, \downarrow \downarrow$, and $\uparrow \downarrow$ and a noncollinear phase (NC) in the absence (a) and presence (b, c) of anisotropy in the easy plane are shown: $b_{1}=b_{2}=0.1$ (b) and $b_{1}=0.2, b_{2}=0$ (c) $\left(b_{i}\right.$ $=\beta_{i} / J_{0}$ are dimensionless anisotropy constants).

expression (8) is meaningful only for $-2<M<2$. In addition, the limited range of the individual functions $\cos \varphi_{i}(-1$ $\left.<\cos \varphi_{i}<1\right)$ imposes additional conditions on the region of acceptable values of the parameters

$$
-1<\frac{4 I^{2} h \pm\left(1-h^{2}\right)^{2}}{2 I\left(1-h^{2}\right)(1+h)}<1 .
$$

The phase diagram of the regions of stability of different configurations of the ferromagnetic part of the system in the $(h, I)$ plane in the case of zero easy-plane anisotropy is presented in Fig. 3a.

The magnetic field dependences of the magnetization of the ferromagnetic layers are presented in Fig. 4 for different ratios of the exchange interaction between the ferromagnetic layers and through the ferro/antiferromagnetic boundary, i.e. different values of the parameter $I$. The dependences for the collinear phases correspond to the horizontal segments of the
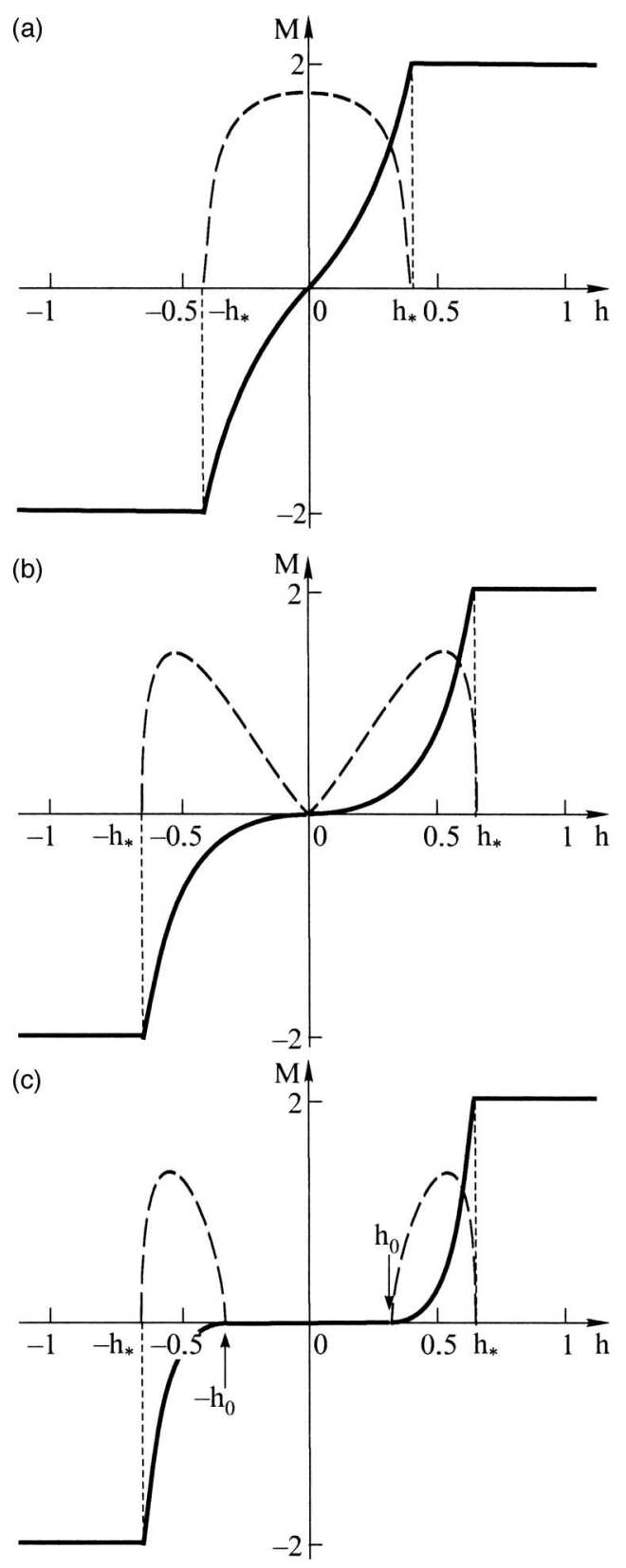

FIG. 4. Longitudinal (solid lines) and transverse (dashed lines) magnetization versus the external field $h$ for different values of the parameter $I$ : a) $I$ $>1 / 2(I=1.1)$, b) $I=1 / 2$, c) $I<1 / 2(I=0.48)$.

straight lines, and the dependences for the noncollinear (canted) phases correspond to the inclined curves. For clarity, the field dependences for perpendicular magnetization $M_{\perp}$ $=\sin \varphi_{1}+\sin \varphi_{2}$ are also presented (dashed curves). For fields in the range $\sqrt{1+2 I}<|h|<\sqrt{1+I^{2}}+I$ there exist additional canted phases, which are, evidently, unstable and are not presented in the figure. Consequently, we shall discuss only collinear and canted structures in the region $|h|<1$.

For $I>1$ the anticollinear structure $\uparrow \downarrow$ with $M=0$ ("small pedestal") is absent, and the canted structure exists in the range $|h|<h_{*}<1$, where the boundary of the interval depends on the parameter $I$ as follows: $h_{*}=\sqrt{1+I^{2}}-I$. For $I$ $=1$ there appears a narrow region of solutions, which is symmetric with respect to $h$, for an anticollinear structure of width $2(1-I)$, which increases in width as $I$ decreases, but these solutions are unstable in the range $1 / 2<I<1$. Figure 

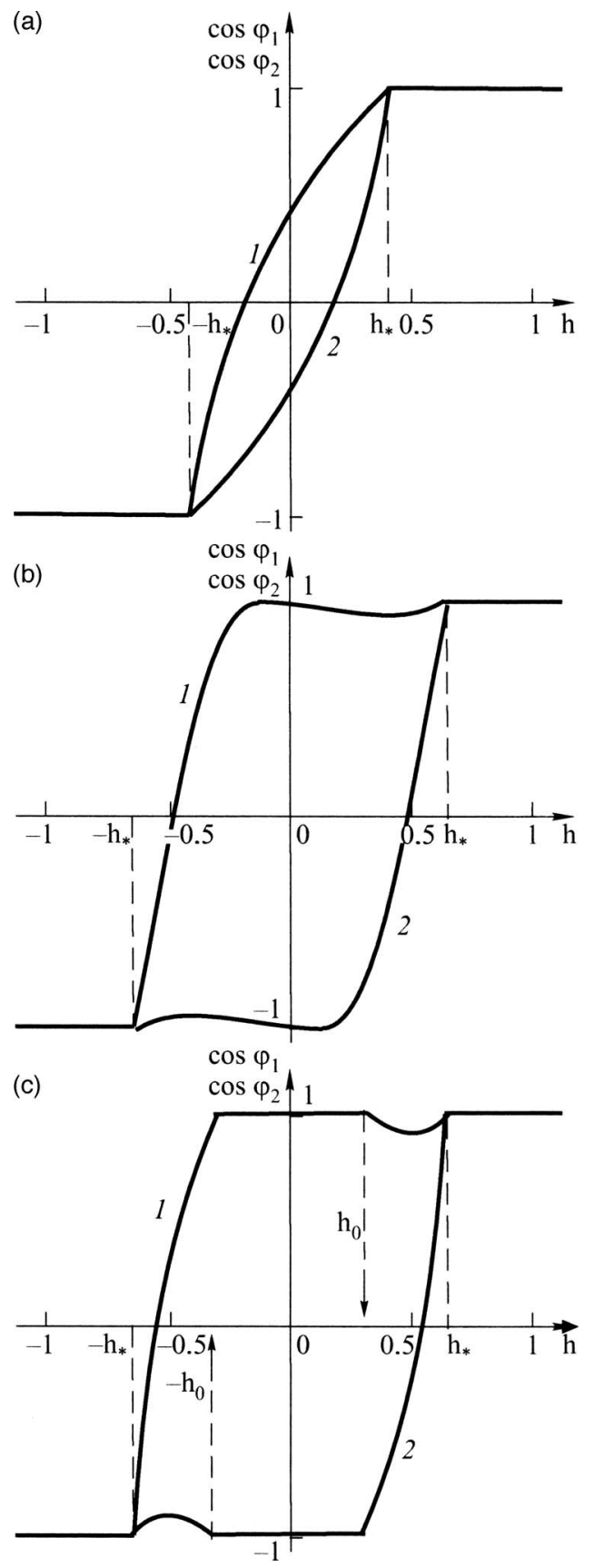

FIG. 5. Field dependences of the $Z$ component of the magnetization of the first (1) and second (2) ferromagnetic layers: a) $I>1 / 2(I=1.1)$, b) $I=1 / 2$, c) $I<1 / 2(I=0.48)$.

4a displays the dependence $M(H)$ for $1 / 2<I<1$, and the corresponding dependence of the $Z$ components of the turning spins in the FM layers are displayed in Fig. 5a. For the canted structure $d M /\left.d h\right|_{h=0}>0$. This derivative vanishes at the critical value $I=1 / 2$ (Fig. $4 \mathrm{~b}$ ): $d M /\left.d h\right|_{h=0}=0$. The corresponding dependences of the magnetization in the layers are displayed in Fig. 5b. Finally, the field dependences of the total magnetization and the magnetizations in the layer with $0<I<1 / 2$ are presented in Figs. $4 \mathrm{c}$ and $5 \mathrm{c}$, respectively. This is the most interesting range of values of the parameter $I$. In this range canted phase exists only for fields $h_{0}<|h|$ $<h_{*}$, where $h_{0}=\sqrt{1-2 I}$, and a solution for the antiparallel phase exists in the range $|h|<1-I$. That is, in the interval $\sqrt{1-2 I}<|h|<h_{*}$ the canted structure coexists with different collinear structures and the stability of different states must be investigated. (At the points $|h|=h_{0}$ the derivative $d M /\left.d h\right|_{h=h \mathrm{o}}=0$.)

\section{STABILITY OF MAGNETIC PHASES WITH DIFFERENT SYMMETRY}

We shall now investigate the stability of different structures in the region $h_{0}<|h|<h_{*}$. The total energy of the system (1) in the notation introduced above is

$\mathcal{E}=\frac{2 E}{J_{0}}=-(h+1) \cos \varphi_{1}-(h-1) \cos \varphi_{2}-I \cos \left(\varphi_{1}-\varphi_{2}\right)$.

In this notation the energies of the collinear (parallel and antiparallel) and canted phases have the form

$$
\begin{aligned}
& \mathcal{E}_{\uparrow \uparrow}=-I-2 h, \quad \mathcal{E}_{\downarrow \downarrow}=-I+2 h, \quad \mathcal{E}_{\uparrow \downarrow}=I-2, \\
& \mathcal{E}_{N C}=\frac{h^{2}-1}{2 I}+\frac{I\left(h^{2}+1\right)}{h^{2}-1} .
\end{aligned}
$$

For $I>1$ only the collinear and canted structures exist, and for $I<1$ a "small pedestal," corresponding to the antiparallel structure and lying higher in energy than the dependence for the canted phase, appears in the dependence $\mathcal{E}(h)$. The field dependences of the energy for different phases are presented in Fig. 6: $I>1$ (a), $I=1 / 2$ (b), and $I<1 / 2$ (c). The energies of the collinear structures are identical to the energy of the canted structure for $|h|=h_{*}$, and at these points $d \mathcal{E}_{N C} / d h=d \mathcal{E}_{\uparrow \uparrow, \downarrow \downarrow} / d h$. The situation is different for $I>1 / 2$ and $I<1 / 2$. For $I>1 / 2$ the energy of the canted phase has its minimum value (less than the energies of the collinear phases) in the entire region of existence of this phase, and the phase is stable. For $I<1 / 2$ a stable anticollinear structure exists in the range $|h|<h_{*}$, and the canted phase does not exist at all. In the range $h_{0}<|h|<h_{*}$ the lowest-energy structure is the canted structure, which is stable. We shall examine the possibility of the existence of metastable states, i.e. we shall examine the stability of all solutions found.

The stability of these solutions is determined by the positive-definiteness of the determinant of the matrix of derivatives $D=\operatorname{det}\left|\partial^{2} \mathcal{E} / \partial \varphi_{i} \partial \varphi_{j}\right|>0$. In order for a state to correspond to minimum energy the condition $K=\partial^{2} \mathcal{E} / \partial \varphi_{1}^{2}>0$ must also be satisfied. This means that the following inequalities must be satisfied simultaneously:

$$
\begin{aligned}
K= & (h+1) \cos \varphi_{1}+I \cos \left(\varphi_{1}-\varphi_{2}\right)>0, \\
D= & \left(h^{2}-1\right) \cos \varphi_{1} \cos \varphi_{2}+I \cos \left(\varphi_{1}-\varphi_{2}\right) \\
& \times\left[(h+1) \cos \varphi_{1}+(h-1) \cos \varphi_{2}\right]>0 .
\end{aligned}
$$

For the noncollinear solutions (7) the condition (13) always holds, since it can be shown that for them $D=I[(1$ $\left.+h) \sin \varphi_{1}\right]^{2}$. However, the first condition (12) can be reduced to the inequality $K=I(1+h) /(1-h)>0$, which holds for $|h|$ $>1$. Consequently, canted structures are unstable for $|h|>0$, as mentioned above. For $|h|<1$ noncollinear solutions exist only in the range $h_{0}<|h|<h_{*}$, where they are stable. For $I$ $>1$ the collinear phases with $|h|>h_{*}$ and the canted phase with $|h|<h_{*}$ correspond to the only energy minima. All other 


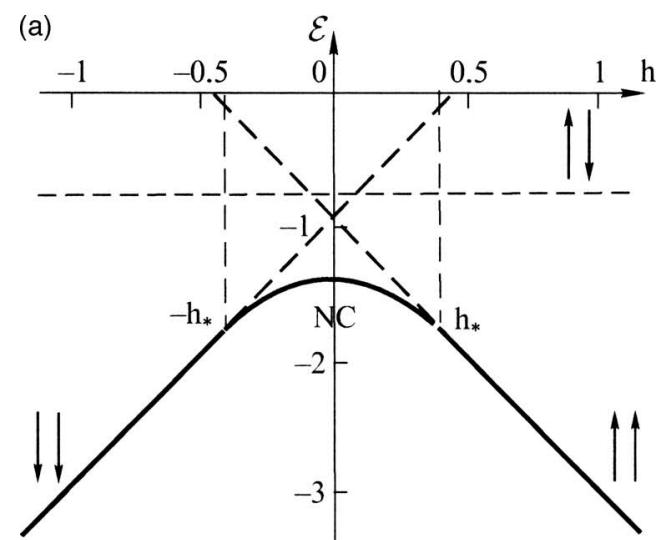

(b)

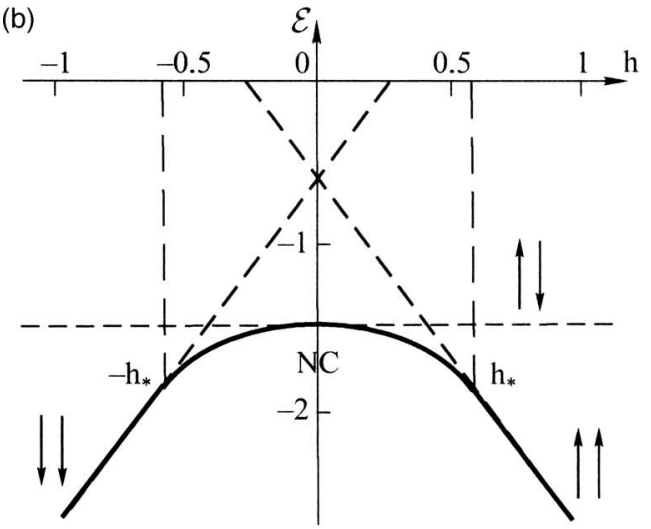

(c)

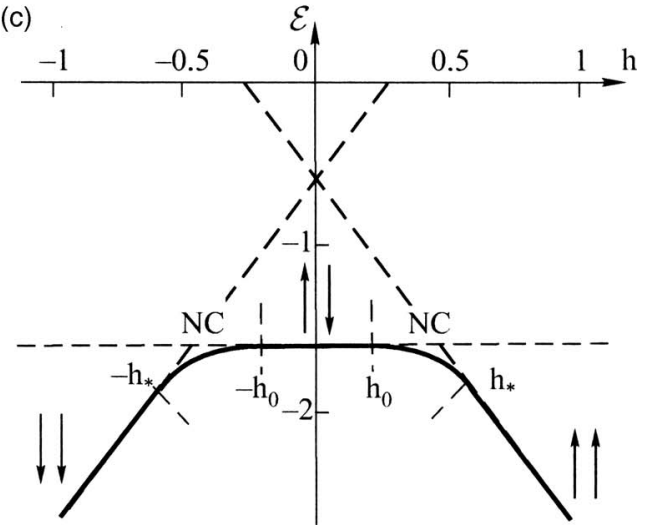

FIG. 6. Field dependences of the energy $\mathcal{E}(h)$ for different values of the parameter $I$ : a) $I>1 / 2(I=1.1)$, b) $I=1 / 2$, c) $I<1 / 2(I=0.48)$. The solid and dashed lines correspond to stable and unstable, respectively, states.

states correspond to saddle points and maxima of the energy $\mathcal{E}\left(\varphi_{1}, \varphi_{2}\right)$ as a function of the angles of the spins. Thus there is no hysteresis in the field dependence of the magnetization. This (absence of relative energy minima) is also true of other ranges of the parameter $I$ : energy minima correspond to only collinear phases for $|h|>h_{*}$, the canted phase for $|h|<h_{*}$ $(I>1 / 2)$ and $h_{0}<|h|<h_{*}(I<1 / 2)$, and the anticollinear phase for $|h|<h_{0}(I<1 / 2)$. In Fig. 3a each point in the parameter plane $(I, h)$ corresponds to one and only one stable phase (there are no additional relative energy minima). Phase transitions proceed at the boundaries of the phases in a continuous manner and hysteresis does not arise in the system. We note that this is valid only if the anisotropy of the ferromagnet is neglected in the initial model.

The final field dependence of the magnetization in the initial variables is as follows. For $J>J_{0} / 4$, there exists a canted structure for negative fields with $H_{-}<H<H_{+}$, where $H_{ \pm}=-J_{0} / 2 \pm\left(\sqrt{4 J^{2}+J_{0}^{2}}-2 J\right) / 2$. The situation is more complicated for $J<J_{0} / 4$ : inside the region $\left(H_{-}, H_{+}\right)$there arises a region $J-J_{0}<H<-J$ where the system is in an anticollinear phase. That is, the magnetization curve is indeed shifted in this range of parameters and possesses a small pedestal; this corresponds to certain elements of the experimental dependences. In the limit $J=0$ the magnetization curve possesses two vertical steps: one at $H=0$ and another at $H=-J_{0}$. This is entirely natural: the ferromagnetic spin layers are coupled with one another, and in zero field the spin (2) flips while in a field $-J_{0}$ the spin (1) closest to the boundary flips.

\section{EFFECT OF MAGNETIC ANISOTROPY IN THE EASY-PLANE ON THE FIELD DEPENDENCES OF THE MAGNETIZATION}

We shall now examine the effect of single-ion magnetic anisotropy in the easy-plane on the properties of magnetization reversal in our simple model. Since this anisotropy is due to the properties of the atoms surrounding the spins it is natural to assume that the anisotropy of the two ferromagnetic layers is different, because the magnetic moments of the ferromagnetic layer (1) are in contact with the atoms of the second ferromagnetic layer and with the atoms of the antiferromagnetic from the contiguous layer. We now introduce the easy-axis anisotropy in the rotation plane of the spins and assume that the anisotropy axis of the ferromagnet is parallel to the anisotropy of the antiferromagnet and the direction of the external field (i.e. it is directed along the $Z$ axis). Here the expression for the energy (1) is modified as follows:

$$
\begin{aligned}
E= & -J_{0} \cos \varphi_{1}-J \cos \left(\varphi_{1}-\varphi_{2}\right)-H\left(\cos \varphi_{1}+\cos \varphi_{2}\right) \\
& -\frac{\beta_{1}}{2} \cos ^{2} \varphi_{1}-\frac{\beta_{2}}{2} \cos ^{2} \varphi_{2} .
\end{aligned}
$$

To obtain a qualitative understanding of the phenomenon we shall examine the simplest case first: the limit $J=0$. In contrast to the preceding case where there is no anisotropy, now this anisotropy results in a possibility of the existence of states with a relative energy minimum, which results in the appearance of hysteresis. It is easy to show that only collinear structures correspond to energy minima (absolute and relative) on the potential energy surface $E=E\left(\varphi_{1}, \varphi_{2}\right)$ and the saddle points of this surface for small anisotropy constants $\left(\beta_{1}=\beta_{2}<J_{0} / 2\right)$ correspond only to collinear structures. (For large anisotropy parameters a minimum corresponding to a canted phase appears, but its energy is higher than that of the all other stable states.)

The transformation of the magnetization curve (for $J$ $=0)$ taking account of the anisotropy is displayed in Fig. 7. The energies $E_{\uparrow \uparrow}$ for $H>0, E_{\uparrow \downarrow}$ for $-J_{0}<H<0$, and $E_{\downarrow \downarrow}$ for $H<-J_{0}$ correspond to an absolute minimum of the energy. The hysteresis curve is symmetric relative to the point $H=-J_{0} / 2=H_{\text {bias }}$ with $\beta_{1}=\beta_{2}$, and for $\beta_{1} \neq \beta_{2}$ asymmetry appears in this dependence. The width of the right-hand loop (centered at $H=0$ ) is $2 \beta_{2}$ and that of the left-hand loop (centered at $\left.H=-J_{0}\right)$ is $2 \beta_{1}$. A small pedestal corresponding to an anticollinear phase lies between these loops (Fig. 7a). The 

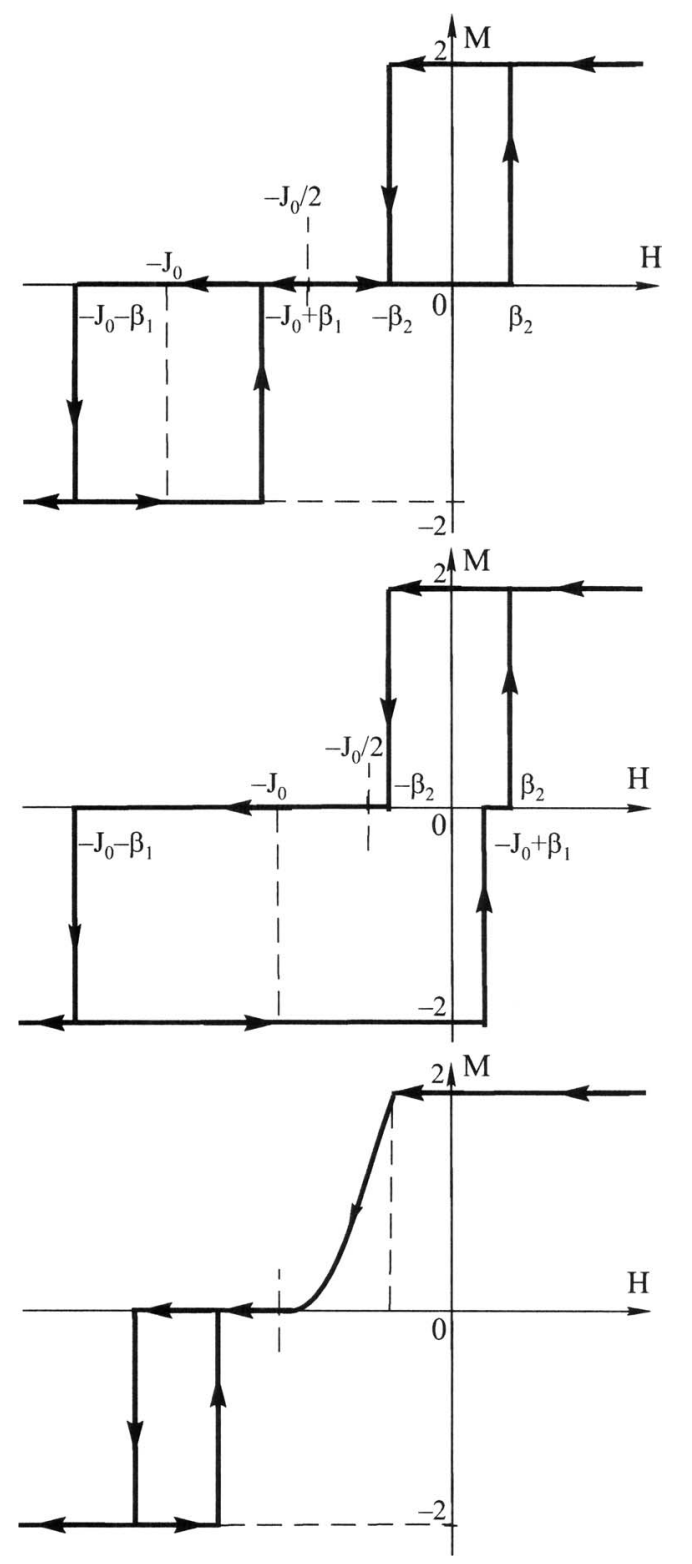

FIG. 7. Magnetization curves for the ferromagnetic subsystem for $J_{0}>\beta_{1}$ $+\beta_{2}, J=0$ (a), $J_{0}<\beta_{1}<J_{0}+\beta_{2}, J=0$ (b), and $\beta_{1} \neq \beta_{2}, J \neq 0$ (c).

transformation of this curve for $J_{0}<\beta_{1}<J_{0}+\beta_{2}$ is interesting (Fig. 7b). In this case the hysteresis curve is reminiscent of the experimental curve in Fig. $1 b$.

We shall now examine the problem briefly, taking account of the anisotropy and nonzero interlayer exchange interaction simultaneously in the ferromagnetic part of the system $(J>0)$. First, we note that for $\beta_{1}=\beta_{2}$ the symmetry property of the hysteresis loop in a field $h$ shifted by the amount of the exchange shift is preserved. For $\beta_{1} \neq \beta_{2}$ this symmetry is broken. When anisotropy is taken into account it is impossible to obtain an analytic solution for the canted phase, but it is possible to find the boundaries of stability for the collinear phases. For this it is necessary to use the inequalities (12) and (13). In addition to the dimensionless notation introduced above for the field and the exchange interaction (6), it is convenient to introduce dimensionless anisotropy constants $b_{i}=\beta_{i} / J_{0}$. The stability boundaries for the various collinear phases now take on the form

$$
\begin{aligned}
& h> h_{\uparrow \uparrow}=-I-b_{1}-b_{2} \\
&+\sqrt{I^{2}+\left(1+b_{1}-b_{2}\right)^{2}} \text { for the phase } \uparrow \uparrow, \\
& h< h_{\downarrow \downarrow}=+I+b_{1}+b_{2} \\
&-\sqrt{I^{2}+\left(1-b_{1}+b_{2}\right)^{2}} \text { for the phase } \downarrow \downarrow, \\
& h_{\uparrow \downarrow}^{1}<h<h_{\uparrow \downarrow}^{2}, \\
& h_{\uparrow \downarrow}^{1,2}=b_{2}-b_{1} \mp \sqrt{\left(1+b_{1}+b_{2}\right)\left(1+b_{1}+b_{2}-2 I\right)} \\
& \quad \text { for the phase } \uparrow \downarrow .
\end{aligned}
$$

As the average anisotropy $\left(b_{1}+b_{2}\right)$ increases, the collinear phases stabilize, i.e. their regions of stability expand and start to overlap. First-order transitions directly between collinear phases without the formation of a canted phase now become possible. The parameter $\left(b_{1}-b_{2}\right)$ characterizes the degree of asymmetry of the transitions. Figures $3 b$ and $3 c$ display phase diagrams of the structural change of the ferromagnetic layers. These diagrams generalize the diagram shown in Fig. 3a to cases of identical anisotropy constants $b_{1}=b_{2}=0.1$ (Fig. 3b) and different constants $b_{1}=0.2, b_{2}=0$ (Fig. 3c). It follows from these figures that in the case of identical anisotropy constants $b_{1}=b_{2}$ regions of canted phases and regions of hysteresis behavior cannot exist simultaneously on the magnetization curve for any value of the exchange $I$. However, for different anisotropy constants $b_{1}$ $\neq b_{2}$ and small values of $I$ (for example, $I_{*}$ in Fig. $3 \mathrm{c}$ ) such phase can exist simultaneously (see Fig. 7c).

\section{CONCLUSION}

A model describing a planar contact between ferro- and antiferromagnets in a magnetic field was proposed. The model describes a ferromagnetic layer consisting only of two magnetic planes. The AFM was assumed to be magnetically rigid (i.e. with fixed directions of the moments in the layer adjoining the interface) and the AFM boundary uncompensated. The strong FM-type magnetic anisotropy of the easymagnetization plane was taken into account, reducing the model to a scalar model. In the proposed model the expression for the exchange bias field $H_{\text {bias }}$ is identical to the expression in the trivial model. ${ }^{4}$ However, the field width of the region of magnetization reversal is finite, which is due to the formation of a noncollinear (canted) phase. The possibility of the existence of canted structures is interesting, because this leads to the existence of inclined sections on the field dependence of the magnetization $M(H)$ which are not associated with the kinetics of magnetization reversal of the ferromagnet. In addition, for small (compared with the exchange interaction $J_{0}$ through the interface) ferromagnetic exchange $J\left(J<J_{0} / 2\right)$ a "small pedestal" with $M=0$ corresponding to the antisymmetric phase $\uparrow \downarrow$ is observed in the field dependence of the magnetization of the ferromagnetic layer near the field $H_{\text {bias }}$. The magnetization curve is strictly symmetric relative to the field $H_{\text {bias }}$. A hysteresis loop appears when the magnetic anisotropy in the easy plane of the FM is taken into account. Anisotropy was taken into account in the limit of very weak FM exchange $(J \rightarrow 0)$ to gain a qualitative understanding of the magnetization reversal process. It was dem- 
onstrated that strong asymmetry of the hysteresis loop obtains when anisotropy is taken into account. The results obtained give a qualitative description of a number of experimentally observed dependences of the magnetization curves (exchange bias; presence of "small pedestals," inclined sections of the curves, and asymmetry of the hysteresis loops).

Partial financial support for this work was provided by a contract for scientific collaboration between NASU (Ukraine) and CNRS (France), a grant from the Academy of Sciences of Ukraine on research into nanostructures, grant N 8-2009 for scientific collaboration between the National Academy of Sciences of Ukraine and RFFI Russia, a grant from the Royal Society of UK (Great Britain), and a grant from the Swedish Academy of Sciences.

a)Email: kovalev@ilt.kharkov.ua

${ }^{1}$ A. G. Grechnev and A. S. Kovalev, Fiz. Nizk. Temp. 24, 340 (1998) [Low Temp. Phys. 24, 257 (1998)].

${ }^{2}$ A. G. Grechnev and A. S. Kovalev, Fiz. Nizk. Temp. 24, 839 (1998) [Low Temp. Phys. 24, 629 (1998)].

${ }^{3}$ A. G. Grechnev and A. S. Kovalev, Fiz. Nizk. Temp. 26, 457 (2000) [Low Temp. Phys. 26, 334 (2000)].

${ }^{4}$ W. H. Meiklejohn and C. P. Bean, Phys, Phys. Rev. 102, 1413 (1956).

${ }^{5}$ J. Nogués and I. K. Schuller, J. Magn. Magn. Mater. 192, 203 (1999).

${ }^{6}$ A. E. Berkowitz and K. Takano, J. Magn. Magn. Mater. 200, 552 (1999).

${ }^{7}$ M. Kiwi, J. Magn. Magn. Mater. 234, 584 (2001).

${ }^{8}$ L. Neel, Ann. Phys. (Paris) 2, 61 (1997).

${ }^{9}$ D. Mauri, H. C. Siegmann, P. S. Bagus, and E. Kay, J. Appl. Phys. 62,
3047 (1987).

${ }^{10}$ M. Kiwi, J. Mejfa-Lopez, R. D. Portugal, and R. Ramirez, Appl. Phys. Lett. 75, 2995 (1999).

${ }^{11}$ M. Kiwi, J. Mejfa-Lopez, R. D. Portugal, and R. Ramirez, Europhys. Lett. 48, 573 (1999).

${ }^{12}$ M. Kiwi, J. Mejfa-Lopez, R. D. Portugal, and R. Ramirez, Solid State Commun. 116, 315 (2000).

${ }^{13}$ J. Mejfa-Lopez, R. Ramirez, and M. Kiwi, J. Magn. Magn. Mater. 241, 346 (2002).

${ }^{14}$ M. D. Stiles and R. D. McMichael, Phys. Rev. B 59, 3722 (1999).

${ }^{15}$ A. P. Malozemoff, Phys. Rev. B 35, 3679 (1987).

${ }^{16}$ T. C. Schulthess and W. H. Butler, J. Appl. Phys. 85, 5510 (1999).

${ }^{17}$ N. C. Koon, Phys. Rev. Lett. 78, 4865 (1997).

${ }^{18}$ M. R. Fitzsimmons, P. C. Yashar, C. Leighton, J. Nogués, J. Dura, C. F. Majkrzak, and J. K. Schuller, Phys. Rev. Lett. 84, 3986 (2000).

${ }^{19}$ M. Gierlings, M. J. Prandolini, H. Fritzsche, M. Gruyters, and D. Riegel, Phys. Rev. B 65, 92407 (2002).

${ }^{20}$ A. Hoffmann, Phys. Rev. Lett. 93, 097203 (2004).

${ }^{21}$ D. N. Merenkov, A. N. Bludov, S. L. Gnatchenko, M. Baran, R. Szymczak, V. A. Hovosad, Fiz. Nizk. Temp. 33, 1260 (2007) [Low Temp. Phys. 33, 957 (2007)].

${ }^{22}$ S. L. Gnatchenko, D. N. Merenkov, A. N. Bludov, V. V. Pishko, Yu. A. Shakhayeva, M. Baran, R. Szymczak, and V. A. Novosad, J. Magn. Magn. Mater. 307, 263 (2006).

${ }^{23}$ D. Spenato, S. P. Pogossian, and H. LeGall, J. Magn. Magn. Mater. 262, 294 (2003)

${ }^{24}$ D. Spenato and S. P. Pogossian, J. Magn. Magn. Mater. 285, 79 (2005).

${ }^{25}$ I. Camarero, J. Sort, A. Hoffmann, J. M. Garcia-Martin, B. Dieny, R. Miranda, and J. Nogués, Phys. Rev. Lett. 95, 057204 (2005).

${ }^{26}$ M. V. Gvozdikova and A. S. Kovalev, Fiz. Nizk. Temp. 24, 1077 (1998) [Low Temp. Phys. 24, 808 (1998)]; M. V. Gvozdikova and A. S. Kovalev, Fiz. Nizk. Temp. 25, 1295 (1999) [Low Temp. Phys. 25, 972 (1999)].

${ }^{27}$ A. S. Kovalev and Ya. E. Prilepskii, Fiz. Nizk. Temp. 29, 71 (2003) [Low Temp. Phys. 29, 55 (2003)].

Translated by M. E. Alferieff 